\section{Which patients will respond to ECT?}

SIR: Scott (Journal, January 1989, 154, 8-17) suggests that a rise in prolactin levels following ECT may be a response to the stress of the ECT procedure, and supports his argument by citing Deakin et al (1983), who showed that simulated ECT increases plasma prolactin concentration twofold. However, what Dr Scott fails to mention in his article is that Deakin et al also showed a sixfold rise in plasma prolactin concentration following real ECT. The difference in the rise of prolactin between the two procedures was statistically significant, which allowed Deakin et al to conclude that the pattern of hormonal changes following ECT are specific to ECT and not simply an effect of stress. The most consistently reported endocrine effect of ECT is a rapid increase in plasma prolactin, and in fact it is so consistent and characteristic that Trimble (1978) suggested that a plasma prolactin increase may be used to differentiate an epileptic fit from hysteria.

With regard to the TRH stimulation test, and its usefulness in identifying depressed patients who will recover with ECT, we would like to draw Dr Scott's attention to Krog-Meyer et al's (1984) study, which reported the results of 39 patients with unipolar depression who recovered after ECT. TRH tests were carried out before and after a course of ECT treatment. All patients received maintenance amitriptyline for 3 weeks, and then were assigned to one of two groups: one group showing an increase in $\triangle \max T S H$ response to TRH greater than $2.0 \mathrm{mIU} / \mathrm{ml}$ after ECT, and the other showing a lesser increase in $\Delta \max$ TSH. The first group and half of the second group received placebo for the next 6 months; the other half of the poor TSH responders received amitriptyline. At the end of 6 months the TSH response tests were repeated, and all patients received no further medication for 6 months. After 6 months, 3 of 15 patients in Group I, and 9 of 13 patients in Group II who received placebo, relapsed, while only 2 of 11 patients in Group II who received amitriptyline relapsed. The authors concluded that failure of TSH response to TRH to normalise is a poor prognostic sign in depressed patients treated with ECT and, furthermore, that maintenance with amitriptyline may prevent relapse.

The amount of cortisol released during the course of ECT may have a relationship to outcome, and in this context we would like to cite Swartz \& Chen (1985), who showed that 10 of the 11 patients who responded to ECT had a progressive fall in the amount of cortisol released during the ECT course, while one non-responder showed an increase in the amount of cortisol released after the last treatment compared with the first.
In relation to EEG studied in ECT, Scott fails to mention the important work of Stromgren \& JuulJensen (1975), who concluded that following a course of ECT, the therapeutic effect of ECT is positively correlated to the incidence and degree of EEG changes. In this study, the importance of obtaining strong universal seizures was emphasised. Failure to elicit such seizures can lead to inefficient and ineffective treatments which prolong the duration of a course.

Finally, regarding seizure threshold, preliminary results of a study we are conducting suggests that although there is a wide variation in baseline seizure threshold, patients who respond to ECT and who maintain this response at six months are those whose seizure threshold rises significantly over the course of their treatment.

Obviously, the whole area needs further exploration and it is difficult to draw any firm conclusions at this stage.

LAKSHMI N. YATHAM SIOBHAN BARRY TIMOTHY G. DiNAN MARCUS WebB

Department of Psychiatry

St James's Hospital

Dublin 8

Ireland

\section{References}

Deakin, J. F. W., Ferrier, I. N., Crow, T. J., et al (1983) Effects of ECT on pituitary hormone release: relationship to seizure, clinical variables and outcome. British Journal of Psychiatry, 143, 618-624

Krog-Meyer, I KirkegaArd, C. Kune, B. et al (1984) Prediction of relapse with the TRH test and prophylactic amitriptyline in 39 patients with endogenous depression. American Journal of Psychiatry, 141, 945-948.

Stromgren, L. S. \& JUUL-Jensen, P. (1975) EEG in unilateral and bilateral electroconvulsive therapy. Acta Psychiatrica Scandinavica, 51, 340-360.

SWARTZ, C. \& ChEN, J. J. (1985) Electroconvulsive therapy induced cortisol release: changes with depressive state. Convulsive Therapy, 1, 15-21.

TRIMBLE, M. R. C. (1978) Serum prolactin in epilepsy and hysteria. British Medical Journal, ii, 1682.

SIR: I was interested to learn of the work of Dr Yatham et al concerning seizure threshold and prolonged recovery after ECT. The correspondents also highlight a few of the many physiological changes that occur during a course of ECT.

My review focused on the everyday clinical problem of predicting which treatment will be best for a particular depressed patient. If the aim is to predict, or foretell, then the observation on which the 
prediction is based must be made before, or at the outset of, treatment. Only one of the measures cited (the release of prolactin) can be made at the first ECT; the other measures can only be made once treatment is complete and cannot be predictive. Prediction of relapse after successful treatment is another important clinical concern, but one I did not cover in my review. Concerning the release of prolactin after ECT, I do not doubt that real ECT produces a greater increase than simulated ECT.

For reasons I have already given in the review, I cannot recommend any of these biological measures to clinicians to improve their ability to select depressed patients who will recover after ECT.

University Department of Psychiatry

Allan Scott

The Kennedy Tower

Royal Edinburgh Hospital

Morningside Park

Edinburgh EHIO 5HF

\section{EEG monitoring of ECT}

SiR: McCreadie et al (Journal, February 1989, 154, 229-231) propose a case for routine EEG monitoring of ECT, particularly for unilateral treatments. However, there are certain flaws in their paper.

Firstly, Dr McCreadie et al have misquoted the findings of Christensen \& Koldbaek (1982). They state that Christensen \& Koldbaek found $43 \%$ of fits inadequate in terms of EEG signs when judged by clinical observation alone. In fact, they reported the opposite: $43 \%$ of fits were inadequate as judged by the clinical method, but only $9 \%$ were inadequate by EEG criteria.

A further misconception concerns the ability of the EEG to demonstrate all seizure activity. Where disagreement arises between the EEG and clinical methods as to whether a seizure has occurred, Dr McCreadie et al unjustifiably assume that the EEG finding is correct and the clinical method false. One type of disagreement (no EEG fit/clinical fit) is interpreted as clinical misdiagnosis of a seizure. However, it is well recognised that seizure activity is not always visible even on a conventional multichannel EEG, let alone the single channel used by the authors. It is questionable whether two bifrontal EEG leads will reliably pick up seizure activity, as the potential difference between the EEG electrodes may be equal in a bilateral seizure. Furthermore, as seizure activity progresses through the cerebral hemispheres, the precise duration of a seizure cannot be demonstrated by a single channel EEG.
The authors state that if only EEG seizures lasting over 25 seconds are counted, disagreement between the EEG and clinical method increases. However, this remark is tautological, since clinical seizures were not measured. In fact, Dr McCreadie et al have shown that, as far as eliciting whether a seizure has occurred is concerned, the EEG and clinical methods agree in the vast majority of cases, with no significant difference between bilateral and unilateral treatments $(97.5 \%$ and $92 \%$ respectively). Thus the conclusion that unilateral ECT requires EEG monitoring seems unwarranted from their data.

Finally, I should like to contribute some points, derived from my own experience (Jones, 1988), to counterbalance the argument that EEG monitoring of ECT is clinically useful.

Firstly, reliable performance of EEG electrodes requires time-consuming preparation of the scalp. In practice, this means that the EEG electrodes and leads have to be attached to the patient prior to anaesthesia. In my experience patients, who are already apprehensive about ECT, find this procedure distressing.

Secondly, frontotemporal EEG electrodes can obstruct the correct application of the ECT electrodes.

Thirdly, mains-powered EEG machines which are not designed for use with ECT are potentially dangerous if so used. This is because, unless the mains connections are electrically isolated, there would be a possibility of the electroshock short-circuiting from a scalp electrode to earth through the EEG machine, which could result in electrocution of the patient. I overcame this problem by adapting a battery operated audible biofeedback machine for monitoring of ECT. Two of the more recently developed ECT devices, the Thymatron and the MECTRA, incorporate a single lead EEG and have circuitry to eliminate electrical errors of this kind.

Fourthly, few junior doctors administering ECT are proficient in reading the EEG. Moreover, preoccupation with the EEG display can detract from their attention to the clinical care of the patient.

Unfortunately, not all anaesthetists are aware of the importance of timing seizure duration in ECT, and therefore sometimes fail to adjust the dose of muscle relaxant during a course of ECT. However, with judicious use of anaesthetic agents, clinical monitoring of seizure duration is usually possible. The case for EEG monitoring of ECT remains unproven.

Department of Mental Health

ERICA M. Jones 